\title{
铬催化苯甲醚衍生物与芳香脂的选择性还原交叉偶联反应
}

\author{
叶 杨 ${ }^{a}$ 龚和贵 $*, a, b$ \\ ( ${ }^{a}$ 上海大学材料科学与工程学院 上海 200444) \\ ( ${ }^{b}$ 上海大学化学系 超分子化学与催化中心 上海 200444)
}

\section{Chromium Catalyzed Reductive Chemoselective Cross-Coupling between Anisole Derivatives and Aryl Ester}

\author{
Ye, Yang ${ }^{a}$ Gong, Hegui*,a,b \\ ( ${ }^{a}$ School of Materials Science and Engineering, Shanghai University, Shanghai 200444) \\ ( ${ }^{b}$ Center for Supramolecular Chemistry and Catalysis, Department of Chemistry, Shanghai University, Shanghai 200444)
}

过渡金属催化的交叉偶联反应是合成化学中化学 键构建的一种强有力的策略 ${ }^{[1]}$. 而联芳烃骨架结构除在 广泛存在于天然化合物及生物活性分子中外，在金属配 体及材料化学中也有重要作用 ${ }^{[2]}$.与过渡金属催化的传 统的芳基亲电试剂与芳基亲核试剂(如有机嗍、有机镁 和有机锌试剂) 的交叉偶联构建 $\mathrm{C}($ aryl $)-\mathrm{C}$ (aryl)键不

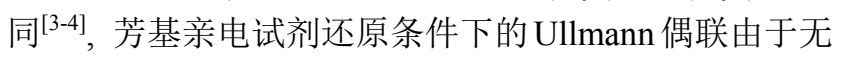
需制备芳基亲核试剂, 因此一直以来是有机合成关注的 研究内容之一. 但是如何有效控制两种不同亲电试剂之 间交叉偶联效率即化学选择性是还原条件下构建 $\mathrm{C}(\operatorname{aryl})-\mathrm{C}(\operatorname{aryl})$ 键的关键挑战 ${ }^{[5]}$. 目前主要的采用的策 略包括采用 $\mathrm{Pd} / \mathrm{Ni}$ 双金属对 $\mathrm{ArOTf}$ 及 $\mathrm{ArBr}$ 的高效偶联, 或利用单个金属如 $\mathrm{Ni}$ 对不同电性的芳基亲电试剂进行 有效偶联 ${ }^{[5,6]}$.

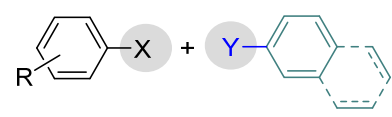

$\mathrm{X}, \mathrm{Y}=$ halides, OTf, OTs, etc.

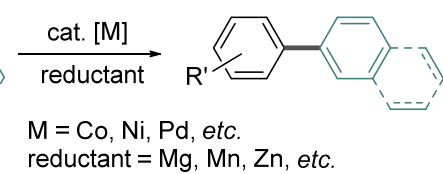

reductant $=\mathrm{Mg}, \mathrm{Mn}, \mathrm{Zn}$, etc.
图 1 过渡金属催化亲电交叉偶联构建 $\mathrm{C}(\operatorname{aryl})-\mathrm{C}(\operatorname{aryl})$ 键 Scheme 1 Transition-metal-catalyzed cross-electrophile coupling to forge $\mathrm{C}($ aryl $)-\mathrm{C}($ aryl $)$ bonds

铬元素是地壳中含量最为丰富的十种元素之一, 虽 然六价态的铬盐具有一定毒性, 但低价态的二氯化铬具 有廉价和无毒的特点. 因此, 以这类低价易得的铬盐为 催化剂前体、探索金属铬独特的反应活性、开发新型的 铬催化合成反应体系来制备功能化合物具有重要的科
学研究意义. 而苯酚衍生物主要来源于富氧的木质纤维 素植物, 与卤代物相比具有天然丰富性和无毒性特点, 是一种常见的芳基亲电试剂[7].

近日，四川大学曾小明团队报道了铬催化苯甲醚衍 生物与芳香酯的选择性交叉偶联反应 ${ }^{[8]}$. 基于该课题组 在探索低价铬催化有机合成反应方面的前期研究结 果 ${ }^{[9]}$, 他们巧妙采用亚胺为邻位导向基团, 使极难参与 氧化加成的甲氧基芳香醚 $\mathrm{C}-\mathrm{O}$ 键可以有效地与施章杰 课题组报道的酰基化的芳基醚 $\mathrm{C}-\mathrm{O}$ 键在还原剂为 $\mathrm{Mg}$ 粉条件下进行还原偶联生成相应的联芳烃产物 ${ }^{[10]}$.

通过大量的反应条件优化实验，他们发现使用 10 $\mathrm{mol} \%$ 的二氯化铬为催化剂、 $10 \mathrm{~mol} \%$ 的 4,4'二叔丁基2,2'-联吡啶(dtbpy)为配体, 3.5 倍当量的镁为还原剂, 在 $40{ }^{\circ} \mathrm{C}$ 条件下可以实现苯甲醚邻位 $\mathrm{C}(\mathrm{aryl})-\mathrm{O}$ 键的高效 萗基化, 而间甲氧基被保留(Scheme 2). 在现有的最佳 条件下，作者首先考察了反应中芳基甲醚底物的适应范 围(Scheme 2), 发现含烷基、烷氧基、苯基、氟等多种 基团取代的苯基甲醚(ArOMe) 都能高效地反应生成二 芳醛，而基团的空间位阻效应对其影响也较小. 此外, 还可以利用该交叉偶联策略修饰具有生物活性的雌酮 骨架化合物. 接着, 作者继续探索芳香酯 $\left(\mathrm{RCO}_{2} \mathrm{Ar} \mathrm{r}^{\prime}, \mathrm{R}=\right.$ $\left.{ }^{t} \mathrm{Bu}, 1-\mathrm{Ad}\right)$ 在交叉偶联反应中的适应性. 发现该体系适 用于多种富电子或缺电子的萗、菲等多环芳基底物. 典 型的取代基团包括甲氧基、三甲基硅基、新戊酰氧基等. 多氟取代的苯基酯及联苯等也能有效偶联生成相应的 双芳基衍生物. 最后, PhO-1-Ad 能够给出 19\%的产率.

\footnotetext{
* Corresponding author. E-mail: hegui_gong@shu.edu.cn. Published online June 9, 2020.
} 


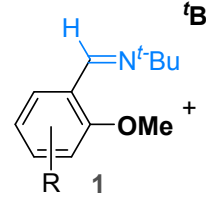

${ }^{t} \mathrm{BuCO}_{2}$

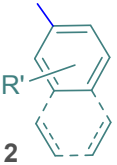

$\mathrm{CrCl}_{2}(10 \mathrm{~mol} \%)$

dtbpy (10 mol\%)

$\mathrm{Mg}$ (3.5 equiv.)

THF, $40^{\circ} \mathrm{C}, 48 \mathrm{~h}$

then $\mathrm{HCl}$ (aq.)
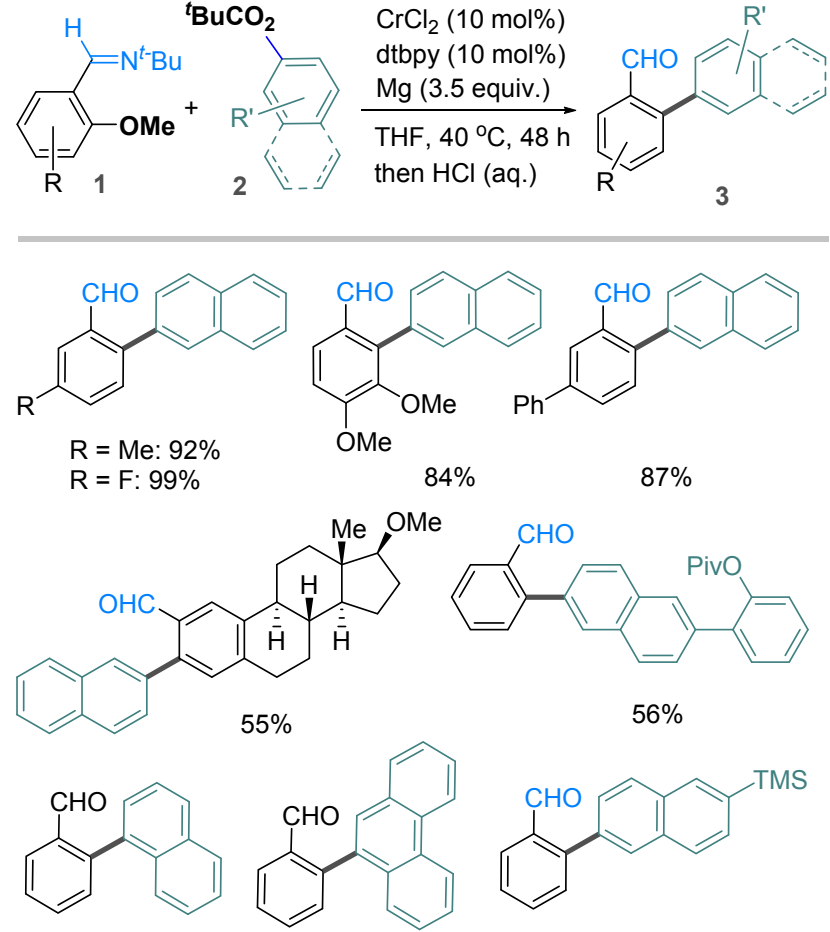

$78 \%$

$88 \%$

$90 \%$

图式 2 反应最优条件和部分底物实例

Scheme 2 Optimized reaction conditions and selected examples

值得一提的是，使用这种亲电交叉偶联策略可用于 1,3-苯并二噁唑衍生物的官能化. 铬可以选择性地将环 $\mathrm{C}($ aryl $)-\mathrm{O}$ 键与非环 $\mathrm{C}($ aryl $)-\mathrm{O}$ 键裂解，并发生开环/ 交叉偶联反应，为制备含邻芳基和间甲醛苯酚化合物提 供了一种新的方法.

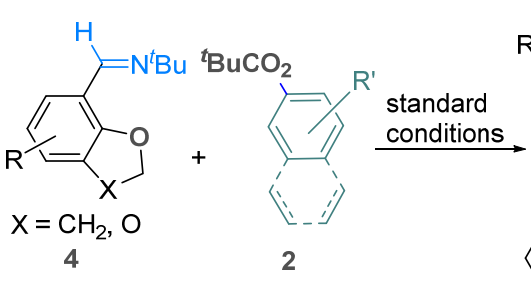

图式 3 开环/交叉偶联反应

Scheme 3 Cr-catalyzed ring-opening/cross-coupling

接着作者进行了一系列机理探究实验. 在 $\mathrm{Cr} / \mathrm{Mg}$ 还 原 $48 \mathrm{~h}$ 后加入化合物 $1 / \mathrm{D}_{2} \mathrm{O}$ 生成少量邻位 C-D 产物的 结果推断, 苯甲醚上的亚胺可能与铬螯合, 从而促进 $\mathrm{C}(\operatorname{aryl})-\mathrm{OMe}$ 键氧化加成生成 $\mathrm{Ar}-\mathrm{Cr}$ 中间体. 而化学 计量控制实验则初步证明了该反应中可能不涉及到格 氏试剂中间产物. 在理论计算(DFT)的进一步支持下, 作者提出了可能的反应机理. 即 $\mathrm{Mg}$ 首先还原 $\mathrm{CrCl}_{2}$ 络
合物生成低价 $\operatorname{Cr}(0)$ ，随后芳基甲醚氧化加成到 $\operatorname{Cr}(0)$ 中 得到芳基 $\mathrm{Cr}$ (II)中间体(IN3), 然后再经过 $\mathrm{Mg}$ 还原和芳 基酯的氧化加成得到的二芳基 $\mathrm{Cr}$ (III)中间体(IN5)，最 后经过还原消除得到交叉偶联产物和 $\mathrm{Cr}(\mathrm{I})$ 中间体. 其 中还原消除为反应的决速步.

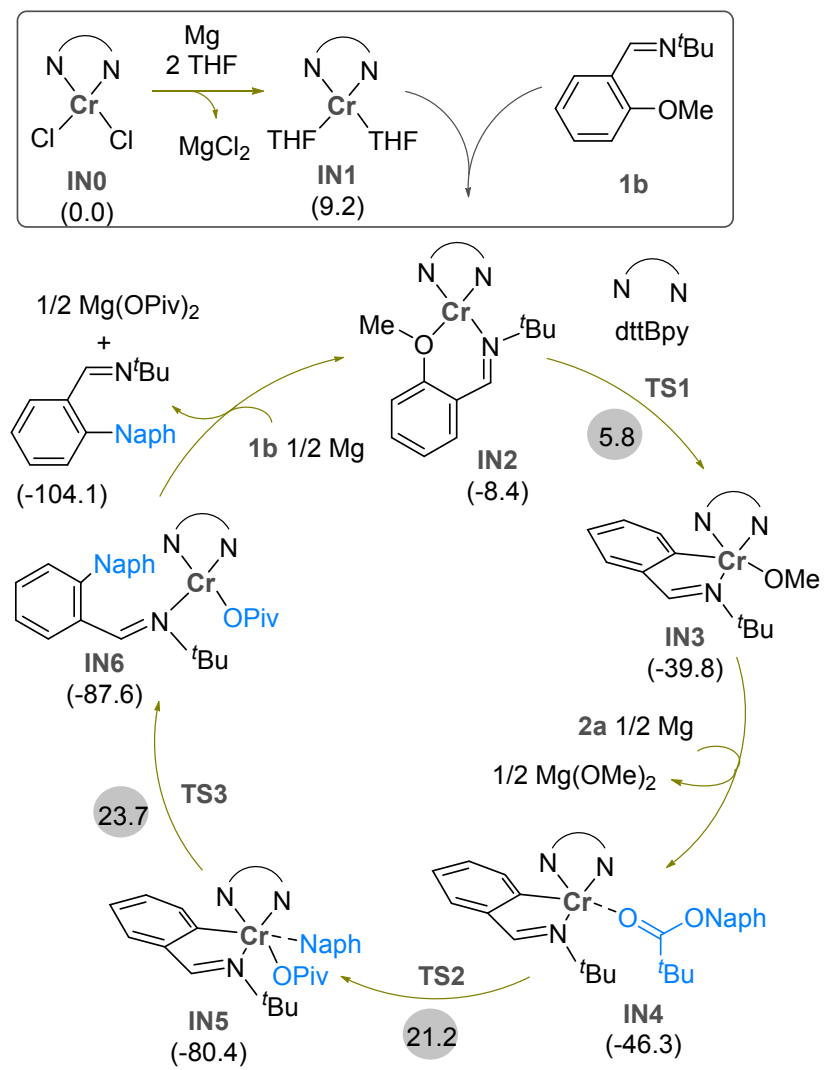

图式 4 DFT 推测的可能机理

Scheme 4 Plausible mechanism identified by DFT calculations

总之, 曾小明团队采用 $\mathrm{Cr} / \mathrm{dtBpy}$ 为新过渡金属催化 剂, $\mathrm{Mg}$ 为还原剂, 在较温和条件下实现了两种不同的非 活化 C(aryl) - $\mathrm{O}$ 键的同步裂解和选择性交叉偶联，开发 了一种新型铬催化的还原 Ullmann 芳基化反应. 对研究 化学键的断裂和重组、设计和开发新型的有机合成反应 具有指导意义.

\section{References}

[1] Negishi, E.-I. Angew. Chem., Int. Ed. 2011, 50, 6738.

[2] Hassan, J.; Sévignon, M.; Gozzi, C.; Schulz, E.; Lemaire, M. Chem. Rev. 2002, 102, 1359.

[3] Miyaura, N.; Suzuki, A. Chem. Rev. 1995, 95, 2457.

[4] Tamao, K.; Sumitani, K.; Kumada, M. J. Am. Chem. Soc. 1972, 94, 4374.

[5] Weix, D. J. Acc. Chem. Res. 2015, 48, 1767.

[6] Amatore, M.; Gosmini, C. Angew. Chem., Int. Ed. 2008, 47, 2089.

[7] Cornella, J.; Zarate, C.; Martin, R. Chem. Soc. Rev. 2014, 43, 8081.

[8] Tang, J.; Liu, L.; Yang, S. Cong, X.; Luo, M.; Zeng, X. J. Am. Chem. Soc. 2020, 142, 7715.

[9] Cong, X.; Tang, H.; Zeng, X. J. Am. Chem. Soc. 2015, 137, 14367.

[10] Yu, D.-G.; Li, B.-J.; Shi, Z.-J. Acc. Chem. Res. 2010, 43, 1486.

(Li, L.) 